\title{
A Novel Index Modulation for Dimming in LiFi Systems
}

\author{
Trang Nguyen, Mohamed Sufyan Islim, and Harald Haas \\ The University of Strathclyde \\ Department of Electrical \& Electronic Engineering \\ LiFi Research and Development Centre \\ Technology \& Innovation Centre, 99 George Street, G1 1RD \\ \{Trang.Nguyen, M.Islim, Harald.Haas\}@ strath.ac.uk
}

\begin{abstract}
This paper introduces a novel dimming method for Light Fidelity (LiFi) based on index modulation (IM). A timedomain sample-index modulation (TIM) is proposed for indexed dimming (iDim). The aim is to maintain a high communication performance measured in signal to noise ratio (SNR) and a high transmission rate for a wide light emitting diode (LED) brightness range. Direct current optical orthogonal frequency division multiplexing (DCO-OFDM) is used. The system performance is experimentally validated by an implementation on a National Instruments (NI) PXIe-1085 and NI-7966R Field Programmable Gate Array (FPGA). The proposed iDim offers a wider dimming range and an improved SNR/symbol when compared to amplitude-modulation dimming (AM dimming). In particular, the iDim system provides a SNR/symbol of $22.5 \mathrm{~dB}$ for all brightness levels. The lowest optical power is measured at $20 \mu \mathrm{W}$ which is 10 times lower than the measured limit of AM dimming. This reduces the cost of the optical power per bit. Therefore, iDim is a promising dimming method for applications targeting extremely low illumination levels.

Index Terms-LiFi, dimming, index modulation, iDim.
\end{abstract}

\section{INTRODUCTION}

We are currently witnessing the commercialization of fifth generation $(5 \mathrm{G})$ mobile networks. Recently, the $6 \mathrm{GHz}$ band was approved for unlicensed WiFi use in the United States by the Federal Communications Commission (FCC) in an aim to meet the exponential growth of the wireless connectivity demand. Moreover, a number of emerging technologies are now being considered for the next wireless generation to future-proof the ever-growing bandwidth demand. Light Fidelity $(\mathrm{LiFi})$ is a promising candidate that has matured after more than a decade of development. The light spectrum is interference-free to the entire radio frequency band and considered to be a green solution in terms of power consumption.

$\mathrm{LiFi}$ aims to use the existing lighting infrastructure for data communications, thus a LiFi light source can serve both

Harald Haas acknowledges the financial support from the Wolfson Foundation and the Royal Society. All authors acknowledge the financial support from the Engineering and Physical Sciences Research Council (EPSRC) under Established Career Fellowship grant EP/R007101/1. illumination and communication purposes. Several technical and functional requirements, such as flicker-free illumination and dimming, are set for existing and ongoing light communication (LC) standards [1] - [3]. The term 'dimming' in LC standards refers to a LiFi system's ability to adjust the transmitter brightness during communications. Dimming is required for LiFi scenarios such as (i) indoor lighting that needs brightness control for user convenience, (ii) aircraft LiFi in which dimmed light is strictly required during flight, and (iii) vehicular laser-based LiFi in which long-range communication (typically over 100 meters) is mandatory while the optical power is restricted by eye safety regulations.

Establishing high performance communication links at low optical power levels is a desired element of this dimming ability. Several dimming methods are available to use. The existing IEEE 802.15.7-2011 standard [1] proposes pulse-widthmodulation (PWM) dimming and amplitude-modulation (AM) dimming methods for rectangular-shaped signals. However, when applying AM dimming and PWM dimming methods to LiFi waveforms, they have shown a degraded performance and a narrow brightness dimming range. In our recent implementation in [4], the AM dimming and the PWM dimming methods are applied on two LiFi modulation schemes, a direct current (DC) optical - orthogonal frequency division multiplexing (DCO-OFDM) and an augmented spectral efficiency - discrete multi-tone (ASE-DMT). The result is summarized in Fig. 1, in which the brightness level (\% unit) is the relative brightness controlled by the transmitter software. It shows that AM dimming has a significant drawback in terms of communication performance when the light is dimmed. Similarly, PWM dimming also shows limitations in terms of the lowest brightness that it can enable, which was measured at $16 \%$ of its maximum.

Moreover, the transmission of any $\mathrm{LiFi}$ waveform requires a certain offset voltage to avoid signal distortion and clipping. In a LiFi dimming scenario, this results in a minimum optical 


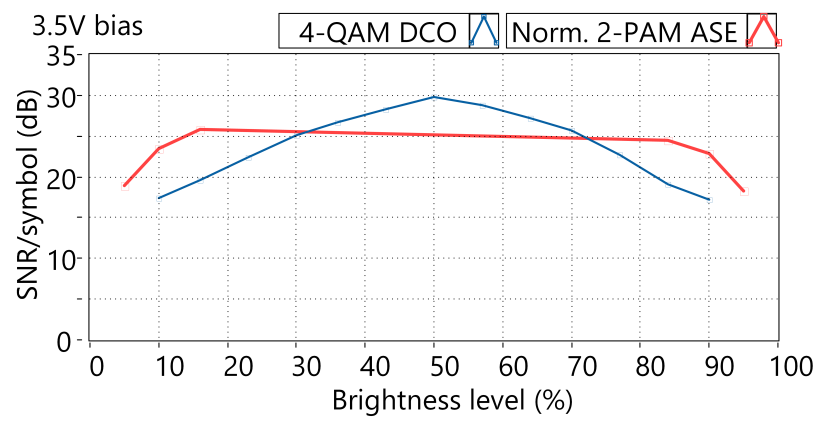

Fig. 1. Measured link performance of ASE-DMT PWM-dimming and DCOOFDM AM-dimming

power that is defined as the $0 \%$ brightness level. However, the $0 \%$ brightness level depends on the selection of the bias voltage. Particularly when selecting bias voltages of $2.85 \mathrm{~V}$, $3 \mathrm{~V}, 3.25 \mathrm{~V}$, and $3.5 \mathrm{~V}$, the $0 \%$ brightness level is measured at around $50 \mu \mathrm{W}, 100 \mu \mathrm{W}, 200 \mu \mathrm{W}$, and $300 \mu \mathrm{W}$, respectively. Thus, even if a dimming level at $0 \%$ brightness is used, the LiFi transmitter will not be entirely off. This limitation can be solved by a novel temporal index modulation (TIM) dimming method, termed indexed dimming (iDim).

The remainder of this paper is organized as follows. Section II introduces the benefits of the proposed iDim scheme by comparing it to few related works. Section III explains the system architecture and implementation guidelines. Section IV explains the implementation of the line codes; and their experimental performance. Moving on, Section V demonstrates the experimental results of the iDim system. Finally, section VI concludes the work.

\section{METHOD AND RELATED WORKS}

\section{A. Related Works}

The concept and performance of index modulation (IM) schemes have recently drawn a lot of interest [5] - [7]. Generally, IM introduces a new dimension for data modulation. Data can be encoded in the space, time and subcarrier index to improve energy efficiency. Most of the IM schemes focus on modulating the indices of subcarriers in subcarrier-index modulation (SIM) [8] - [11]. Applying SIM and spatial modulation to optical communication has been widely considered in recent years [11] - [14].

Until this date, the number of experimental studies on IM is modest. Similarly, even though there are numerous studies related to methodologies for LiFi dimming [15], the number of implementation studies is still few. A notable PWM-inspired dimming method for multi-carrier waveforms was proposed in [16]. It could significantly extend the dimming range by inserting inactive slots and controlling the dimming based on the PWM dimming duty cycle. However, the drawback of this method is a dramatically reduced transmission rate. The proposed iDim has the same PWM-dimmable capability as [16]. However, it gains additional spectral efficiency due to the modulated index bits. More importantly, to the best of our knowledge, the proposed iDim is the first implementation of an efficient index modulation technique designed for $\mathrm{LiFi}$ dimming.

\section{B. iDim Scheme}

1) General benefits of TIM for iDim: The application of IM to $\mathrm{LiFi}$ has been widely considered to improve spectral efficiency [5] - [14]. We propose a novel application of IM for LiFi dimming, in which TIM is chosen for several reasons. First, TIM is more suitable for single light emitting diode (LED) transmission since dimming codes are applied in the time domain. This makes TIM applicable to light sources with single and multiple LEDs. Second, a TIM encoder/decoder operates entirely in the time domain. Thus, the TIM encoder can be used at the output of any OFDM encoder. Moreover, TIM uses a simple real-time encoding/decoding technique while at the same time providing dimming abilities.

2) Transmission rate: In the proposed iDim system both the TIM encoder and OFDM encoder carry data bits. The sum bit rate is normalized in (1) to allow for the comparison between the transmission rate of iDim and DCO-OFDM. The normalized transmission rate varies based on the optical power imposed by the dimming parameter $p$ (i.e., the weight of the binary line code).

$$
R=p \log _{2}\left(N_{\mathrm{QAM}}\right)+2(x / z),
$$

where $p<1$ denotes the dimming parameter; $\log _{2}($.$) denotes$ the binary logarithm; $N_{\mathrm{QAM}}$ is the QAM modulation order; and $x / z$ denotes the rate of the line code $x \mathrm{~B} z \mathrm{~B}$.

\section{PROPOSED SYSTEM}

The hardware configuration for the iDim DCO-OFDM implementation is illustrated in Fig. 2. It is based on a National Instruments (NI) real-time processing platform, PXIe-1085 computer, which is equipped with field programmable gate array (FPGA) NI-7966R panels. An analog-to-digital/ digitalto-analog (ADC/DAC) board (NI-5781, $20 \mathrm{MHz}$ communication bandwidth) operates in the baseband to allow for intensity modulation with direct detection. An alternative current (AC)coupled Photodiode (PD) (Femto-HSPR-X-I-1G4-SI, the 3-dB bandwidth of $1.4 \mathrm{GHz}$ ), and an off-the-shelf LED (Vishay VLMB1500) are used. An additional programmable DC generator (Keysight E36313A) is connected to the DC input of the bias-T to supply the LED with a controllable bias voltage.

The block diagram for the transmitter (TX) is illustrated in Fig. 3. Based on the selected dimming level set by the 
dimming parameter $p$, the TIM encoder loads a code from the lookup table (LUT). The LUT generation is detailed in Algorithm 1. Based on that code, the TIM encoder maps its data bits to decide the indices of the active OFDM samples and inactive samples within the time-domain frame. The inactive samples refer to the samples where the LED is turned off.

Particularly when a line code, $x \mathrm{~B} z \mathrm{~B}$, is loaded, the TIM encoder takes $(p z)$ OFDM samples as an input and inserts $(1-p) z$ inactive samples, where $p<1$. Thus, there will be $z$ samples at the output of the TIM encoder. The index dimension which represents the indices of the active OFDM samples allows us to encode an additional $x$ data bits.

The block diagram for the receiver (RX) is illustrated in Fig. 4. The TIM decoder is located after the synchronization and cyclic prefix removal blocks and before the FFT block, and it operates entirely in the time domain as detailed in Algorithm 2.

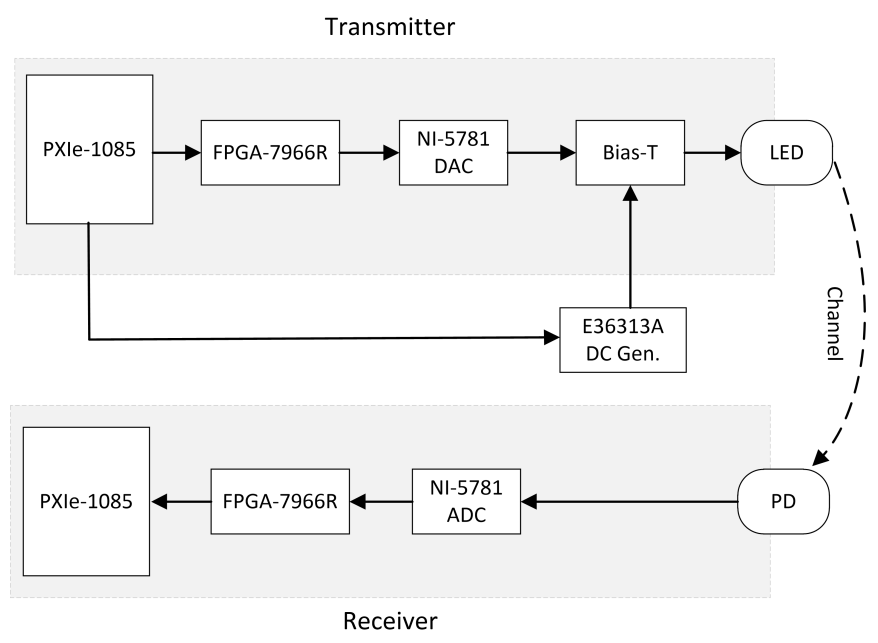

Fig. 2. iDim- DCO-OFDM implementation architecture

\section{Performance of Line Coding}

\section{A. Simulation Performance}

A novel set of line codes are proposed to enable dimming in TIM. The codes 2B4B, 3B8B, and 4B16B are introduced and their performance is evaluated. These codes are simply generated and decoded as given in Algorithms 1 and 2. To easily understand the LUT mapping rule, Table I presents an example of the $2 \mathrm{~B} 4 \mathrm{~B}$ code. In the mapping, the binary ' 1 ' represents an OFDM sample slot while ' 0 ' represents an inactive sample slot where the LED is turned off.

A matched filter (MF) is proposed to maximize the decoder performance. The design of the MF allows us to integrate all codes within the same system.

The MF design significantly improves the bit error rate (BER) performance of the proposed system. The simulation
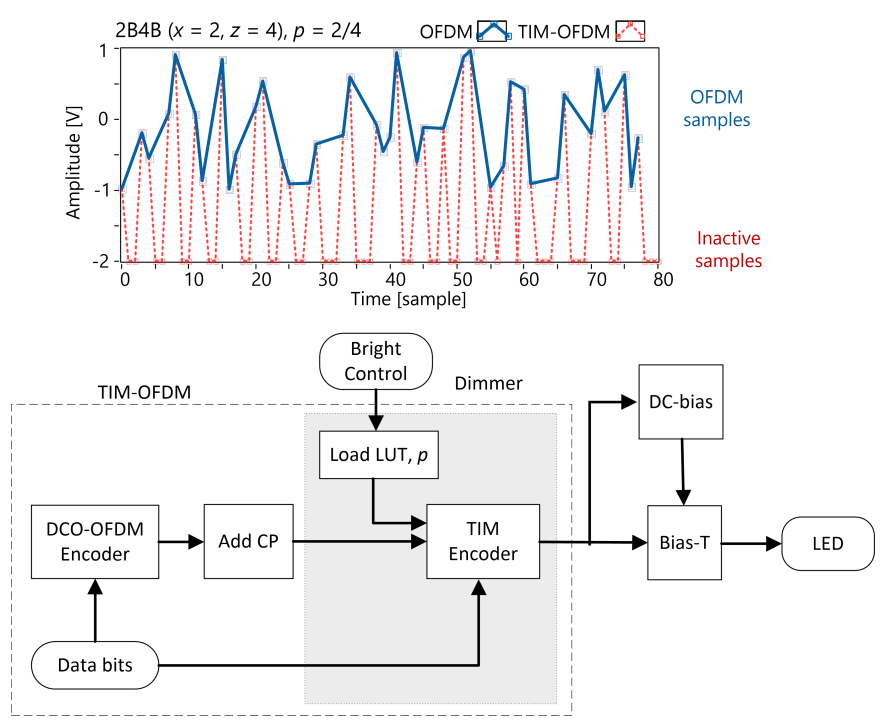

Fig. 3. iDim- DCO-OFDM Transmitter block diagram (bottom), and an example of output waveform when a $2 \mathrm{~B} 4 \mathrm{~B}$ code is used (top)

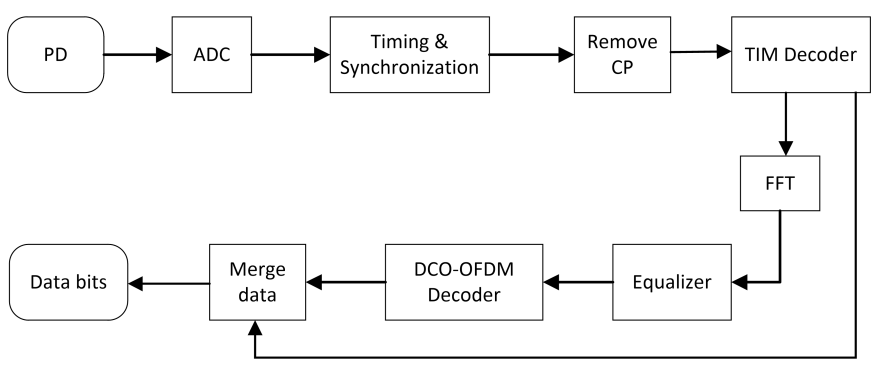

Fig. 4. iDim- DCO-OFDM Receiver block diagram

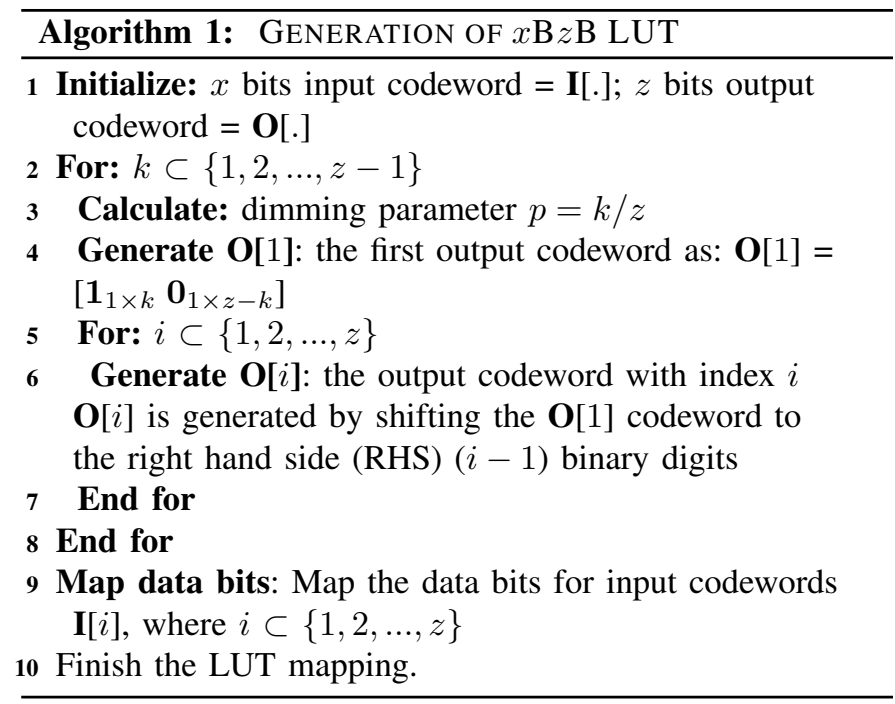




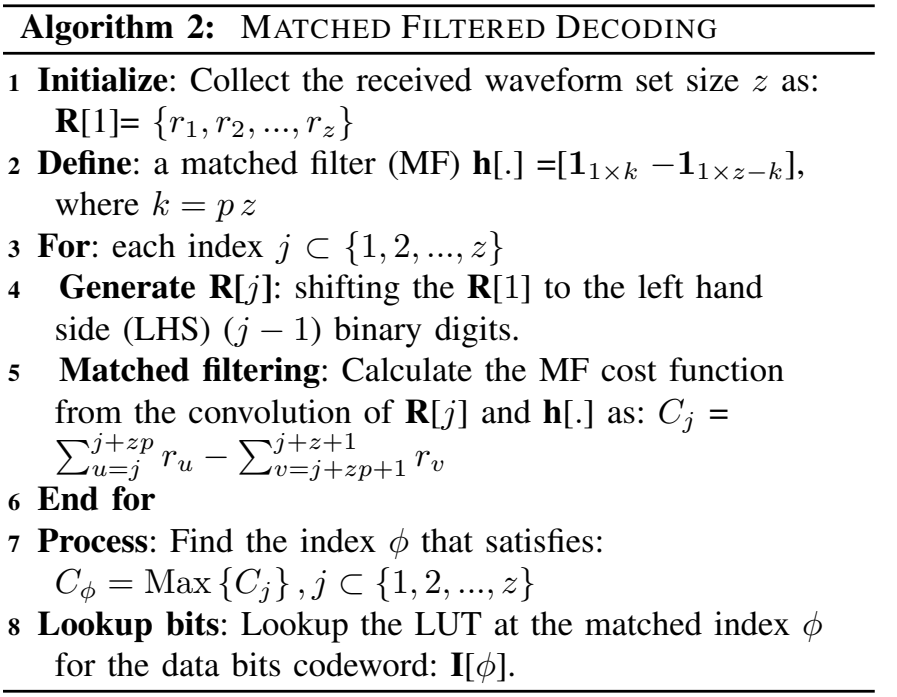

TABLE I

EXAMPLE OF 2B4B LOOKUP TABLE (LUT)

\begin{tabular}{|l|l|l|l|}
\hline 2B Input & $\begin{array}{l}4 \mathrm{~B} \text { Output } \\
(\mathrm{p}=1 / 4)\end{array}$ & $\begin{array}{l}4 \mathrm{~B} \text { Output } \\
(\mathrm{p}=2 / 4)\end{array}$ & $\begin{array}{l}\text { 4B Output } \\
(\mathrm{p}=3 / 4)\end{array}$ \\
\hline 00 & 1000 & 1100 & 1110 \\
\hline 01 & 0100 & 0110 & 0111 \\
\hline 10 & 0010 & 0011 & 1011 \\
\hline 11 & 0001 & 1001 & 1101 \\
\hline
\end{tabular}

results are shown in Figs. 5 and 6 for 2B4B and 3B8B, respectively. The results show that the proposed codes require $3 \mathrm{~dB}$ to $5 \mathrm{~dB}$ less SNR than OOK to achieve the same BER. This was achieved due to the use of the MF.

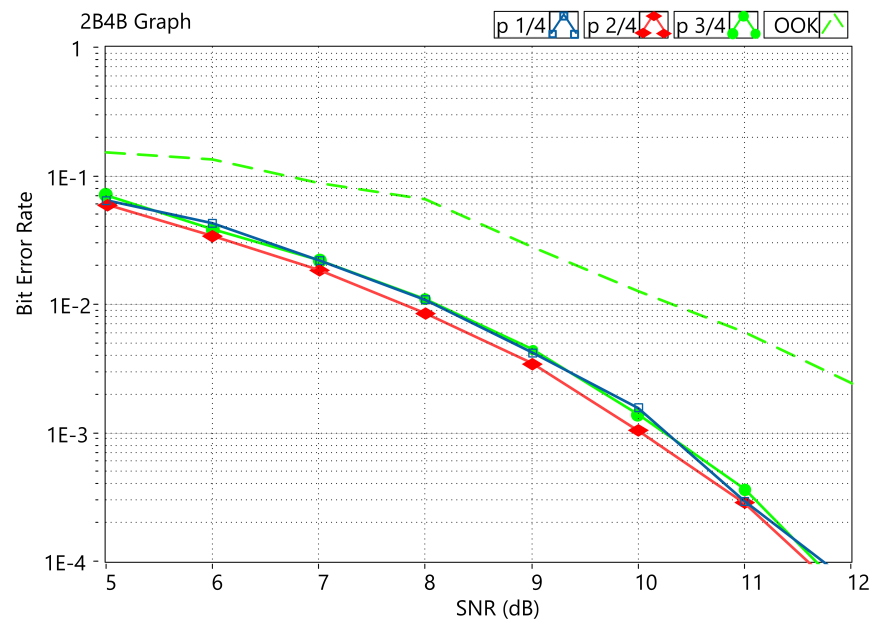

Fig. 5. Matched filtered 2B4B versus OOK Bit Error Rates

The performance of different line codes is examined under different dimming conditions by comparing the performance of the same code and applying a different value for the dimming parameter $p$. The code $2 \mathrm{~B} 4 \mathrm{~B}$ achieves almost the

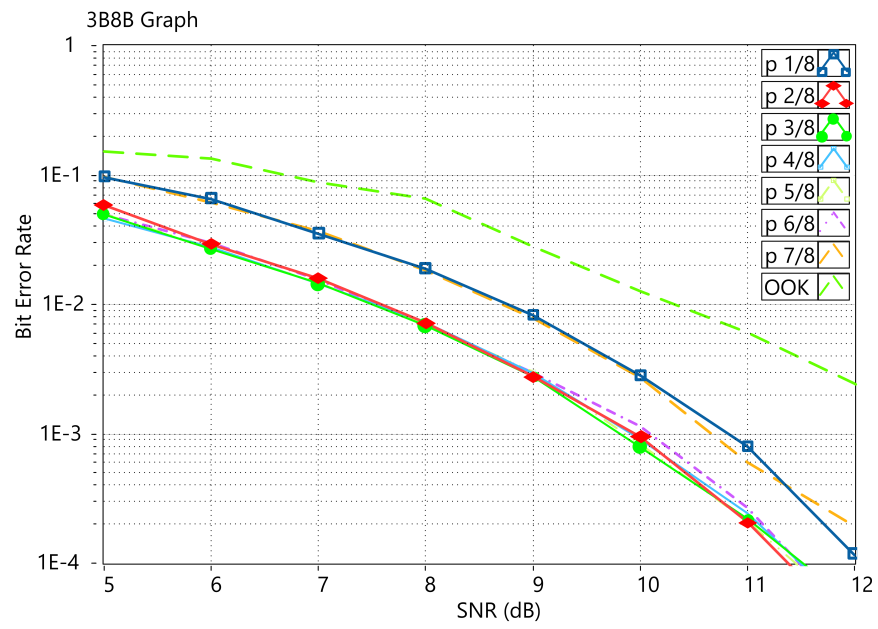

Fig. 6. Matched filtered 3B8B versus OOK Bit Error Rates

same BER performance for all the considered dimming levels. Codes $3 \mathrm{~B} 8 \mathrm{~B}$ and $4 \mathrm{~B} 16 \mathrm{~B}$ have $1 \mathrm{~dB}$ difference between the codes corresponding to the lowest/highest brightness levels and the rest of the codes. Particularly within 3B8B, the BER performance at $p=1 / 8$ is exactly the same as that at $p=7 / 8$; and both require $1 \mathrm{~dB}$ more SNR than the remaining values of $p$ (i.e., $p=2 / 8$ to $p=6 / 8$ ) to reach the same BER. This is due to the unequal average hamming distance between the line codes at different values for $p$. Although the MF gain at $p$ $=1 / 8$ and $p=7 / 8$ is less than it gains at other values of $p$, the lowest performance case of 3B8B (with MF) still outperforms that of OOK.

\section{B. Optical Link Performance}

Two goals were achieved in the implementation of iDim DCO-OFDM. First, the measured optical power reaches an extremely low value $(20 \mu \mathrm{W})$. Second, the SNR/symbol performance of both TIM and DCO-OFDM decoders is high for all dimming levels (above $17 \mathrm{~dB}$ ). This ensures a reliable communication performance for the proposed iDim system during dimming.

The optical power is measured using the optical power meter PM100USB and the S121C photodiode power sensor. Fig. 7 presents the measured optical power for AM-dimming and iDim. The minimum achievable optical power (without any data communications) of the AM-dimming scheme is about $200 \mu \mathrm{W}$ and $90 \mu \mathrm{W}$ in the cases of $3.25 \mathrm{~V}$ and $3 \mathrm{~V}$ bias voltages, respectively. On the other hand, iDim achieves an optical power that is about $20 \mu \mathrm{W}$ at $p=1 / 8$ for all bias voltages. This is a 10 times and 4.5 times optical power reduction, respectively.

The communications performance is evaluated by measuring the SNR-per-symbol (denoted as SNR/symbol or $\gamma$ ) [17]. 


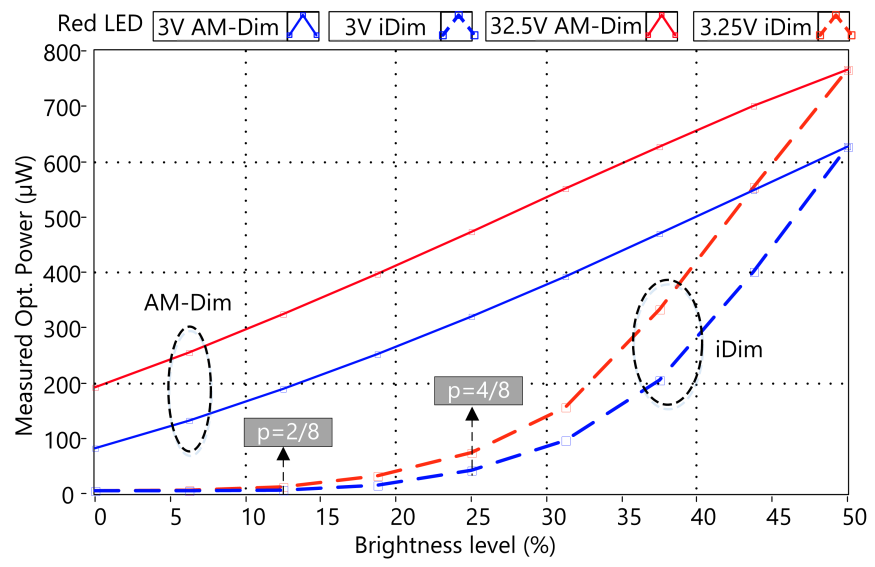

Fig. 7. Measurement of optical power when applying AM-dimming and iDim to DCO-OFDM

The $\mathrm{SNR} / \mathrm{symbol}$, in $\mathrm{dB}$, of an $M$-QAM, where $M$ is the constellation size, is expressed as:

$$
\gamma=20 \log \left(\frac{u_{\mathrm{QAM}}}{\sigma}\right)
$$

where $u_{\mathrm{QAM}}$ denotes the minimum Euclidean distance of the considered QAM order, and $\sigma$ denotes the error vector magnitude (EVM) of the received QAM symbols.

The measurement of the SNR/symbol in the optical links is shown in Fig. 8. Bias voltages of $3 \mathrm{~V}$ and $3.25 \mathrm{~V}$ are both considered. The TIM decoder shows a consistently high performance for both bias voltage cases. More particularly, an SNR/symbol above $20 \mathrm{~dB}$ can be achieved for all optical power levels above $40 \mu \mathrm{W}$ (equivalent to $p$ levels above 4/8). Overall, the TIM achieves a high SNR performance at an extremely low optical power of $40 \mu \mathrm{W}$.

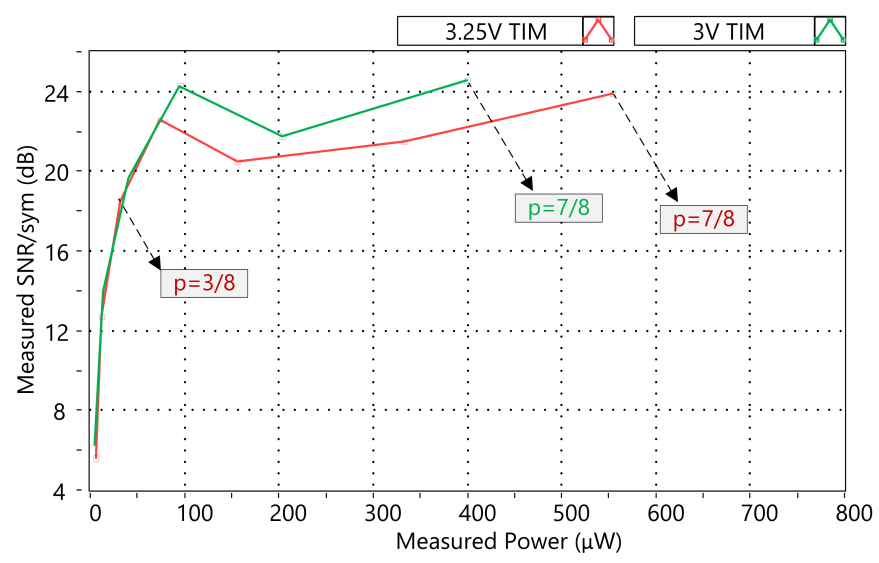

Fig. 8. Measured performance of TIM decoder in iDim DCO-OFDM system ( $3 \mathrm{~V}$ and $3.25 \mathrm{~V}$ bias voltage)

\section{Performance of DCO-OFDM DeCoder In iDim}

\section{A. Optical Link Quality}

This section compares the performance of the DCO-OFDM decoder in the AM-dimming system and our iDim system. Both bias voltages of $3 \mathrm{~V}$ and $3.25 \mathrm{~V}$ are considered. Fig. 9 shows the SNR/symbol measurement results. The 4-QAM modulation order is chosen for DCO-OFDM for comparison purposes. It is also possible to use other constellation sizes.

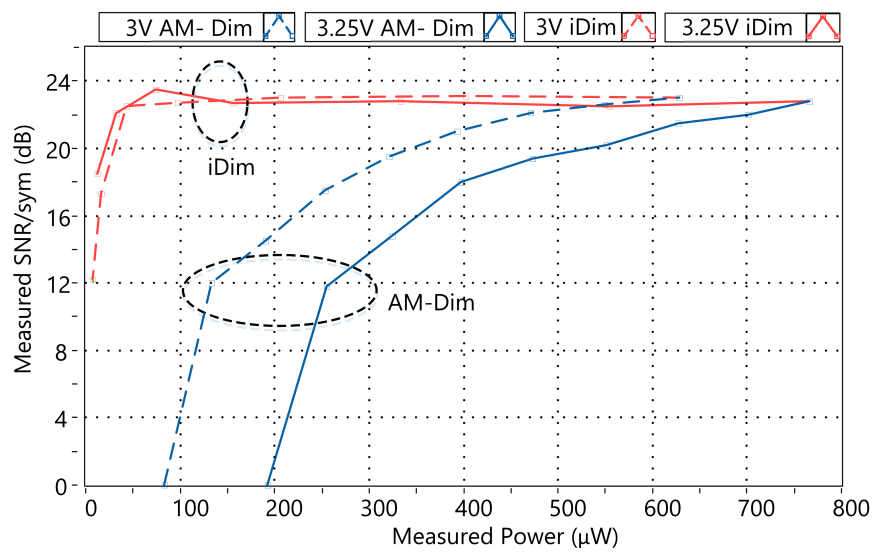

Fig. 9. Measured performance of iDim DCO-OFDM versus AM-dimming DCO-OFDM ( $3 \mathrm{~V}$ and $3.25 \mathrm{~V}$ bias voltage)

At $3 \mathrm{~V}$ bias voltage, the performance of the iDim DCOOFDM system is constant at $23 \mathrm{~dB}$ for all dimming levels above $p=4 / 8$. As $p$ goes lower, the measured optical power drops below $40 \mu \mathrm{W}$ and the corresponding performance starts to degrade (see Fig. 9). The lowest optical power that can guarantee a proper decoding of bits (defined as SNR/symbol above $17 \mathrm{~dB}$ ) is measured at $20 \mu \mathrm{W}$. This is significantly lower than the lowest optical power required for the DCO-OFDM decoder of the AM-Dim system that is given at $200 \mu \mathrm{W}$. Together with the performance measurement of the TIM decoder, we can conclude that the proposed iDim technique has achieved a consistently high performance during dimming, and reached an extremely low optical power. Moreover, it is important to note that the iDim technique provides a high data rate, resulting in an increased energy efficiency.

At $3.25 \mathrm{~V}$ bias voltage, the superior performance of iDim is more pronounced. The AM-dimming dissipates at least $350 \mu \mathrm{W}$ optical power at SNR/symbol values above $15 \mathrm{~dB}$. In comparison, the proposed iDim decoders guarantee a high SNR performance of $18 \mathrm{~dB}$ at an optical power measured at $20 \mu \mathrm{W}$ (17.5 times lower).

\section{B. Energy Efficiency}

The optical power per bit is calculated and presented in Fig. 10 based on the ratio of the measured optical power and the calculated transmission rate in (1). The proposed 
iDim shows a lower optical power/bit for all brightness levels. Moreover, it showcases the ability to reach extremely low optical power values. Particularly, at $130 \mu \mathrm{W}$, AM-dimmed DCO-OFDM requires $66 \mu \mathrm{W} / \mathrm{bit}$ while 2B4B iDim uses $55 \mu \mathrm{W} / \mathrm{bit}$, saving $17 \%$ of the energy. For any brightness below $130 \mu \mathrm{W}$, AM-dimmed DCO-OFDM cannot guarantee a reliable performance below $12 \mathrm{~dB}$ SNR/symbol, while iDim maintains a $22.5 \mathrm{~dB} \mathrm{SNR} / \mathrm{symbol}$ and linearly reduces the required optical power/bit. This results in a dual gain in the achieved SNR/symbol and optical power/bit requirement.

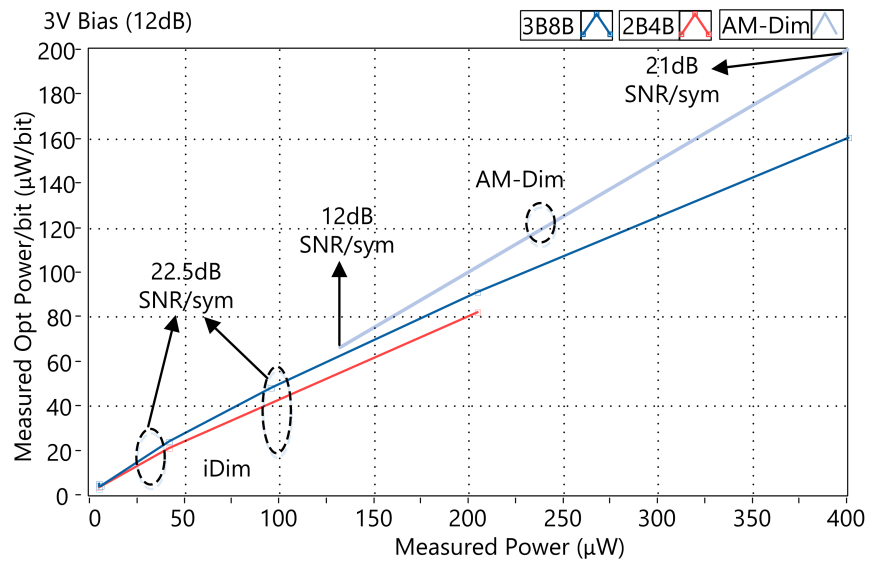

Fig. 10. Measured optical power/bit comparison between AM-Dim and iDim systems ( $3 \mathrm{~V}$ bias voltage)

\section{CONCLUSION}

Novel dimming line codes are proposed and evaluated. A matched filter is designed for TIM decoding to achieve a high BER performance. Despite their variable dimming parameter and unequal code length, their reliable link quality has been proven through simulations and implementations.

The integration of the TIM and DCO-OFDM encoders demonstrates a proof of concept of the indexed dimming method, iDim. The measurements of optical power and the SNR/symbol at both the TIM decoder or the DCO-OFDM decoder show the advantages of iDim. SNR/symbol values above $20 \mathrm{~dB}$ are consistently measured over a wide dimming range. This proves the robust operation of TIM and OFDM decoders in the iDim system. The measurements also show an extremely low optical power/bit performance.

In summary, the benefits of $\mathrm{iDim}$ have been proved through various measurements, including a high SNR/symbol of 22.5 $\mathrm{dB}$, a wide dimming range (any brightness above $20 \mu \mathrm{W}$ ) and a high transmission rate when compared to DCO-OFDM. The joint spectral and energy efficiency is achieved as a result of its low optical power/bit requirements.

\section{REFERENCES}

[1] IEEE Standards Association, "IEEE Standard for Local and Metropolitan Area Networks - Part 15.7: Short-Range Wireless Optical Communication Using Visible Light," Sept. 2011.

[2] IEEE Standards Association, "IEEE 802.15 WPAN Task Group 13 (TG13) Multi-Gigabit/s Optical Wireless Communications". [Online]. Available: http://www.ieee802.org/15/pub/TG13.html.

[3] IEEE Standards Association, "IEEE 802.11 - Light Communication (LC) Task Group (TG) - Meeting update". [Online]. Available: http://www.ieee802.org/11/Reports/tgbb update.htm.

[4] T. Nguyen, M. S. Islim and H. Hass, "Integration of Dimming into LiFi Systems," IEEE 91st Vehicular Technology Conference (VTC2020Spring), Antwerp, Belgium, 2020, pp. 1-5, doi: 10.1109/VTC2020Spring48590.2020.9128398.

[5] E. Basar, M. Wen, R. Mesleh, M. Di Renzo, Y. Xiao and H. Haas, "Index Modulation Techniques for Next-Generation Wireless Networks," in IEEE Acess, vol. 5, pp. 16693-16746, 2017. doi: 10.1109/ACCESS.2017.2737528

[6] X. Cheng, M. Zhang, M. Wen and L. Yang, "Index Modulation for 5G: Striving to Do More with Less," in IEEE Wireless Communications, vol. 25, no. 2, pp. 126-132, Apr. 2018. doi: 10.1109/MWC.2018.1600355

[7] E. Basar, "On Multiple-Input Multiple-Output OFDM with Index Modulation for Next Generation Wireless Networks," in IEEE Transactions on Signal Processing, vol. 64, no. 15, pp. 3868-3878, Aug.1, 2016. doi: 10.1109/TSP.2016.2551687

[8] N. Ishikawa, S. Sugiura and L. Hanzo, "Subcarrier-Index Modulation Aided OFDM - Will It Work?," in IEEE Access, vol. 4, pp. 2580-2593, 2016. doi: 10.1109/ACCESS.2016.2568040

[9] E. Başar, "OFDM With Index Modulation Using Coordinate Interleaving," in IEEE Wireless Communications Letters, vol. 4, no. 4, pp. 381384, Aug. 2015. doi: 10.1109/LWC.2015.2423282

[10] B. Zheng, M. Wen, E. Basar and F. Chen, "Multiple-Input MultipleOutput OFDM With Index Modulation: Low-Complexity Detector Design," in IEEE Transactions on Signal Processing, vol. 65, no. 11, pp. 2758-2772, Jun.1, 2017. doi: 10.1109/TSP.2017.2673803

[11] F. Halabi, L. Chen, R. P. Giddings, A. Hamié and J. M. Tang, "Multilevel Subcarrier Index-Power Modulated Optical OFDM With Adaptive Bit Loading for IMDD PON Systems," in IEEE Photonics Journal, vol. 8, no. 6, pp. 1-14, Dec. 2016, Art no. 7907114. doi: 10.1109/JPHOT.2016.2627624

[12] A. Yesilkaya, E. Basar, F. Miramirkhani, E. Panayirci, M. Uysal and H. Haas, "Optical MIMO-OFDM With Generalized LED Index Modulation," in IEEE Transactions on Communications, vol. 65, no. 8, pp. 3429-3441, Aug. 2017. doi: 10.1109/TCOMM.2017.2699964

[13] R. Y. Mesleh, H. Haas, C. W. Ahn, and S. Yun, "Spatial modulationOFDM," in Proc. Int. OFDMWorkshop, pp. 288 -92, Aug. 2006.

[14] P. Yang, M. Di Renzo, Y. Xiao, S. Li, and L. Hanzo, "Design guidelines for spatial modulation," in IEEE Communications Surveys and Tutorials, vol. 17, no. 1, pp. 6-26, 1st Quart., 2015.

[15] Hany Elgala and Thomas D. C. Little, "Reverse polarity optical-OFDM (RPO-OFDM): dimming compatible OFDM for gigabit VLC links," in Opt. Express 21, 24288-24299, 2013.

[16] G. Ntogari, T. Kamalakis, J. Walewski and T. Sphicopoulos, "Combining Illumination Dimming Based on Pulse-Width Modulation With Visible-Light Communications Based on Discrete Multitone", in J. Opt. Commun. Netw., vol. 3, pp. 56-65, 2011.

[17] N. Robertson, "Compute Modulation Error Ratio (MER) for QAM," Nov. 2019. [Online]. Available: https://www.dsprelated.com/showarticle/1305.php 